Bull. Korean Math. Soc. 48 (2011), No. 5, pp. 1105-1109

http://dx.doi.org/10.4134/BKMS.2011.48.5.1105

\title{
APPROXIMATION AND INTERPOLATION IN THE SPACE OF CONTINUOUS FUNCTIONS VANISHING AT INFINITY
}

\author{
MÁrCia Kashimoto
}

\begin{abstract}
We establish a result concerning simultaneous approximation and interpolation from certain uniformly dense subsets of the space of vector-valued continuous functions vanishing at infinity on locally compact Hausdorff spaces.
\end{abstract}

\section{Introduction and preliminaries}

Throughout this paper we shall assume, unless stated otherwise, that $X$ is a locally compact Hausdorff space and $(E,\|\cdot\|)$ is a normed vector space over $\mathbb{K}$, where $\mathbb{K}$ denotes either the field $\mathbb{R}$ of real numbers or the field $\mathbb{C}$ of complex numbers. We shall denote by $E^{*}$ the topological dual of $E$ and by $C(X ; E)$ the vector space over $\mathbb{K}$ of all continuous functions from $X$ into $E$.

A continuous function $f$ from $X$ to $E$ is said to vanish at infinity if for every $\varepsilon>0$ the set $\{x \in X:\|f(x)\| \geq \varepsilon\}$ is compact. Let $C_{0}(X ; E)$ be the vector space of all continuous functions from $X$ into $E$ vanishing at infinity and equipped with the supremum norm. The vector subspace of all functions in $C(X ; E)$ with compact support is denoted by $C_{c}(X ; E)$.

Let $A$ be a nonempty subset of $C_{0}(X ; \mathbb{K})$. We denote by $A \otimes E$ the subset of $C_{0}(X ; E)$ consisting of all functions of the form

$$
f(x)=\sum_{i=1}^{n} \phi_{i}(x) v_{i}, \quad x \in X,
$$

where $\phi_{i} \in A, v_{i} \in E, i=1, \ldots, n, n \in \mathbb{N}$.

A subset $W \subset C_{0}(X ; E)$ is an interpolating family for $C_{0}(X ; E)$ if given any nonempty finite subset $S \subset X$ and any $f \in C_{0}(X ; E)$, there exists $g \in W$ such that $g(x)=f(x)$ for all $x \in S$.

A nonempty subset $B$ of $C_{0}(X ; E)$ is said to have the approximation-interpolation property on finite subsets (in short, the $S A I$ property) if for every $f \in C_{0}(X ; E)$, every $\varepsilon>0$ and every nonempty finite subset $S$ of $X$, there exists $g \in B$ such that $\|f-g\|<\varepsilon$ and $f(x)=g(x)$ for all $x \in S$.

Received June 1, 2010; Revised August 13, 2010.

2010 Mathematics Subject Classification. Primary 41A65; Secondary 41A05.

Key words and phrases. simultaneous approximation and interpolation, Walsh's theorem. 
The purpose of this paper is to present a result of simultaneous approximation and interpolation from certain subsets of $C_{0}(X ; E)$. As a consequence, we obtain a generalization of a result by Prolla concerning simultaneous approximation and interpolation from vector subspaces of $C(X ; E)$ when $X$ is a compact Hausdorff space.

\section{Main result}

Walsh (Theorem 6.5.1 [2]) proved the following result.

Theorem 2.1. Let $K$ be a compact set in the complex plane and let $z_{1}, \ldots, z_{n}$ be any set of $n$ points in $K$. If the function $f$ is defined on $K$ and can be uniformly approximated by polynomials there, then $f$ can be uniformly approximated by polynomials $p$ which also satisfy the auxiliary conditions $p\left(z_{i}\right)=f\left(z_{i}\right)$, $i=1, \ldots, n$.

Motivated by Walsh, we establish the result below.

Theorem 2.2. Let $A$ be an interpolating family for $C_{0}(X ; \mathbb{K})$ and $B$ an uniformly dense subset of $C_{0}(X ; E)$. If $(A \otimes E)+B \subset B$, then $B$ has the $S A I$ property.

Lemma 2.1. If $X$ is a locally compact Hausdorff space and $\left\{x_{1}, \ldots, x_{n}\right\} \subset X$, then there exists $l_{i} \in C_{c}(X ; \mathbb{R})$ such that $l_{i}\left(x_{i}\right)=1$ and $l_{i}\left(x_{j}\right)=0, j \neq i$.

Proof. Since $X$ is Hausdorff and $\left\{x_{1}, \ldots, x_{n}\right\}$ is finite there exists an open neighborhood $U_{i}$ of $x_{i}$ such that $x_{j} \notin U_{i}$ for all $j \neq i, j \in\{1, \ldots, n\}$. By Urysohn's Lemma [8] there exists $l_{i} \in C_{c}(X ; \mathbb{R}), 0 \leq l_{i} \leq 1$, such that $l_{i}\left(x_{i}\right)=1$ and $l_{i}(x)=0$ if $x \notin U_{i}$, in particular, $l_{i}\left(x_{j}\right)=0, j \neq i$.

Proof of Theorem 2.2. Let $S=\left\{x_{1}, \ldots, x_{n}\right\}$ be a subset of $X$. Let $f \in C_{0}(X ; E)$ and $\varepsilon>0$.

By Lemma 2.1, for each $x_{i} \in S$ there exists $l_{i} \in C_{c}(X ; \mathbb{R})$ such that

$$
\begin{aligned}
& l_{i}\left(x_{i}\right)=1 \\
& l_{i}\left(x_{j}\right)=0 ; j \neq i, x_{j} \in S .
\end{aligned}
$$

Since $A$ is an interpolanting family for $C_{0}(X ; \mathbb{K})$, there exist $\phi_{1}, \ldots, \phi_{n} \in A$ such that

$$
\phi_{i}\left(x_{j}\right)=l_{i}\left(x_{j}\right) ; \quad 1 \leq i, j \leq n .
$$

Since $B$ is uniformly dense in $C_{0}(X ; E)$ there exists $g \in B$ such that $\|f-g\|<$ $\eta$ where $\eta:=\varepsilon /\left(1+\sum_{i=1}^{n}\left\|\phi_{i}\right\|\right)$.

The function $h: X \rightarrow E$ defined by

$$
h(x)=\sum_{i=1}^{n} \phi_{i}(x)\left(f\left(x_{i}\right)-g\left(x_{i}\right)\right)
$$

belongs to $A \otimes E$ and $h\left(x_{j}\right)=f\left(x_{j}\right)-g\left(x_{j}\right)$ for $j=1, \ldots, n$. 
Now the function $p=h+g$ belongs to $B$ and $p\left(x_{j}\right)=f\left(x_{j}\right)$ for $j=1, \ldots, n$. Moreover,

$$
\|f-p\| \leq\|f-g\|+\|h\|<\eta+\eta \sum_{i=1}^{n}\left\|\phi_{i}\right\|=\varepsilon .
$$

Example 2.1. The set of all continuous real-valued nowhere differentiable functions on $[a, b]$, denoted by $N D[a, b]$, has the SAI property. Indeed, let $P[a, b]$ be the set of all real polynomials on $[a, b]$. Note that

(a) $P[a, b]$ is an interpolating subset of $C([a, b] ; \mathbb{R})$ (take the Lagrange polynomials);

(b) $N D[a, b]$ is uniformly dense in $C([a, b] ; \mathbb{R})$;

(c) $(P[a, b] \otimes \mathbb{R})+N D[a, b]=P[a, b]+N D[a, b] \subset N D[a, b]$.

Hence, it follows from Theorem 2.2 that $N D[a, b]$ has the SAI property.

Lemma 2.2. Every uniformly dense vector subspace of $C_{0}(X ; \mathbb{K})$ is an interpolating family for $C_{0}(X ; \mathbb{K})$.

Proof. Let $S=\left\{x_{1}, \ldots, x_{n}\right\}$ be a subset of $X$ and $G$ be an uniformly dense vector subspace of $C_{0}(X ; \mathbb{K})$. Consider the following continuous linear mapping

$$
\begin{aligned}
T: C_{0}(X ; \mathbb{K}) & \rightarrow \mathbb{R}^{n} \\
f & \mapsto\left(f\left(x_{1}\right), \ldots, f\left(x_{n}\right)\right) .
\end{aligned}
$$

Note that $T(G)$ is closed because it is a vector subspace of $\mathbb{R}^{n}$. Then by density of $G$ and continuity of $T$, it follows that

$$
T\left(C_{0}(X ; \mathbb{K})\right)=T(\bar{G}) \subset \overline{T(G)}=T(G) .
$$

Therefore, for any $f \in C_{0}(X ; \mathbb{K})$, there exists $g \in G$ such that $\left(f\left(x_{1}\right), \ldots, f\left(x_{n}\right)\right)$ $=\left(g\left(x_{1}\right), \ldots, g\left(x_{n}\right)\right)$.

A subset $M$ of $C_{0}(X ; \mathbb{K})$ is dense-lineable or algebraically generic if $M \bigcup\{0\}$ contains a vector space dense in $C_{0}(X ; \mathbb{K})$. For more information, see [1].

Corollary 2.1. If $M$ is a dense-lineable subset of $C_{0}(X ; \mathbb{K})$, then $M \bigcup\{0\}$ has the $S A I$ property. In particular, all dense vector subspaces of $C_{0}(X ; \mathbb{K})$ have the SAI property.

Proof. Since $M \bigcup\{0\}$ contains a vector space $A$ dense in $C_{0}(X ; \mathbb{K})$, it follows from Lemma 2.2 that $A$ is an interpolating family for $C_{0}(X ; \mathbb{K})$. Moreover,

$$
(A \bigotimes \mathbb{K})+A \subset A \text {. }
$$

Then, by Theorem 2.2, it follows that $A$ has the SAI property. Since $A \subset$ $M \bigcup\{0\}$ we conclude that $M \bigcup\{0\}$ has the SAI property.

The last corollary can also be proved by using Deutsch's result [3].

In order to give a criterion to identify vector subspaces of $C_{0}(X ; E)$ which have the SAI property, we need the next two results. 
Proposition 2.1. The vector subspace $C_{0}(X ; \mathbb{K}) \otimes E$ is uniformly dense in $C_{0}(X ; E)$.

Proof. It follows from Corollary $6.4[5]$ that $C_{c}(X ; \mathbb{K}) \otimes E$ is uniformly dense in $C_{0}(X ; E)$. Since

$$
C_{c}(X ; \mathbb{K}) \bigotimes E \subset C_{0}(X ; \mathbb{K}) \bigotimes E \subset C_{0}(X ; E),
$$

we conclude that $C_{0}(X ; \mathbb{K}) \otimes E$ is uniformly dense in $C_{0}(X ; E)$.

Lemma 2.3. If $A$ is an uniformly dense subset of $C_{0}(X ; \mathbb{K})$, then $A \otimes E$ is uniformly dense in $C_{0}(X ; E)$.

Proof. By Proposition 2.1, $C_{0}(X ; \mathbb{K}) \otimes E$ is uniformly dense in $C_{0}(X ; E)$. Then given $f \in C_{0}(X ; E)$ and $\varepsilon>0$, there exists $g \in C_{0}(X ; \mathbb{K}) \otimes E$, say $g(x)=\sum_{i=1}^{n} \psi_{j}(x) v_{j}, \psi_{j} \in C_{0}(X ; \mathbb{K}), v_{j} \in E, j=1, \ldots, n$, such that $\|f-g\|<$ $\varepsilon / 2$.

Since $A$ is uniformly dense in $C_{0}(X ; \mathbb{K})$, there exists $a_{j} \in A$ such that

$$
\left\|\psi_{j}-a_{j}\right\|<\frac{\varepsilon}{2\left(\sum_{j=1}^{n}\left\|v_{j}\right\|+1\right)} .
$$

The function $h:=\sum_{j=1}^{n} a_{j} v_{j} \in A \otimes E$. Moreover,

$$
\begin{aligned}
\|f-h\| \leq\|f-g\|+\|g-h\| & <\frac{\varepsilon}{2}+\sum_{j=1}^{n}\left\|\psi_{j}-a_{j}\right\|\left\|v_{j}\right\| \\
& <\frac{\varepsilon}{2}+\frac{\varepsilon}{2\left(\sum_{j=1}^{n}\left\|v_{j}\right\|+1\right)} \sum_{j=1}^{n}\left\|v_{j}\right\|<\varepsilon .
\end{aligned}
$$

We obtain the following result.

Theorem 2.3. If $A$ is an uniformly dense vector subspace of $C_{0}(X ; \mathbb{K})$ and $B$ is a vector subspace of $C_{0}(X ; E)$ such that $A \otimes E \subset B$, then $B$ has the $S A I$ property.

Proof. It follows from Lemma 2.2, Lemma 2.3 and Theorem 2.2.

Corollary 2.2 (Prolla [7], Theorem 7). Let $X$ be a compact Hausdorff space and $B \subset C(X ; E)$ a vector subspace such that $A:=\left\{\phi \circ g: \phi \in E^{*}, g \in B\right\}$ is uniformly dense in $C(X ; \mathbb{K})$ and $A \otimes E \subset B$. Then $B$ has the SAI property.

Example 2.2. Let $(X, d)$ be a compact metric space. A function $f: X \mapsto E$ is called Lipschitzian if there is some constant $K_{f}>0$ such that

$$
\|f(x)-f(y)\| \leq K_{f} d(x, y)
$$

for every $x, y \in X$. We denote by $\operatorname{Lip}(X ; E)$ the subset of $C(X ; E)$ of all such functions. By Theorem $9[6]$, the vector subspace $\operatorname{Lip}(X ; \mathbb{K})$ is uniformly 
dense in $C(X ; \mathbb{K})$. For any $f_{1}, \ldots, f_{n} \in \operatorname{Lip}(X ; \mathbb{K}), v_{1}, \ldots, v_{n} \in E$, there exist constants $k_{1}, \ldots, k_{n}>0$ such that

$$
\left\|\sum_{j=1}^{n} f_{j}(x) v_{j}-f_{j}(y) v_{j}\right\| \leq \sum_{j=1}^{n} \mid f_{j}(x)-f_{j}(y)\left\|v_{j}\right\| \leq\left(\sum_{j=1}^{n} k_{j}\left\|v_{j}\right\|\right) d(x, y)
$$

for every $x, y \in X$. Hence, $\operatorname{Lip}(X ; \mathbb{K}) \otimes E \subset \operatorname{Lip}(X ; E)$. Then, by Theorem 2.3, $\operatorname{Lip}(X ; E)$ has the SAI property.

Example 2.3. Since $C_{c}(X ; \mathbb{K})$ is uniformly dense in $C_{0}(X ; \mathbb{K})$ (see Nachbin [4], p. 64) and $C_{c}(X ; \mathbb{K}) \otimes E \subset C_{c}(X ; E)$, it follows from Theorem 2.3 that $C_{c}(X ; E)$ has the SAI property.

Example 2.4. Let $X_{i}$ be a locally compact Hausdorff space for $i=1, \ldots, n$ and $X=X_{1} \times \cdots \times X_{n}$.

Let $A$ be the set of all finite sums of functions of the form

$$
x=\left(x_{1}, \ldots, x_{n}\right) \mapsto f(x)=g_{1}\left(x_{1}\right) \cdots g_{n}\left(x_{n}\right),
$$

where $g_{j} \in C_{0}\left(X_{j} ; \mathbb{K}\right)$ for $j=1, \ldots, n$. By the weighted Dieudonné theorem ([4] Theorem 1 p. 68), $A$ is an uniformly dense vector subspace of $C_{0}(X ; \mathbb{K})$. From Theorem 2.3, $A \otimes E$ has the SAI property.

Acknowledgement. We thank the referee for comments and suggestions which greatly improved the presentation of the paper.

\section{References}

[1] L. Bernal-González, Dense-lineability in spaces of continuous functions, Proc. Amer. Math. Soc. 36 (2008), no. 9, 3163-3169.

[2] P. J. Davis, Interpolation and Approximation, Dover Publications, New York, 1975.

[3] F. Deutsch, Simultaneous Interpolation and Approximation in Linear Topological Spaces, SIAM J. Appl. Math. 14 (1966), 1180-1190.

[4] L. Nachbin, Elements of Approximation Theory, Van Nostrand, Princeton, NJ, 1967; reprinted by Krieger, Huntington, NY, 1976.

[5] J. B. Prolla, Approximation of Vector-Valued Functions, Mathematics Studies 25, NorthHolland, Amsterdam, 1977.

[6] _ Weierstrass-Stone, The Theorem, Verlag Peter Lang, Frankfurt, 1993.

[7] On the Weierstrass-Stone theorem, J. Approx. Theory 78 (1994), no. 3, 299-313.

[8] W. Rudin, Real and Complex Analysis, McGraw-Hill, Singapore, Third Edition, 1987.

Departamento de Matemática e Computação

Universidade Federal de Itajubá - UNIFEI

Caixa Postal 50

37500-903 Itajubá, MG, BraZiL

E-mail address: kaxixi@unifei.edu.br, mskashim@gmail.com 